The Astrophysical Journal Supplement Series, 90:729-734, 1994 February

(C) 1994. The American Astronomical Society. All rights reserved. Printed in U.S.A.

\title{
ELECTRON-BEAM EVOLUTION IN PARTIALLY IONIZED HYDROGENIC PLASMA WITH RETURN CURRENTS
}

\author{
D. V. SYNIAVSKII ${ }^{1}$ AND V. V. ZHARKOVA ${ }^{1,2}$ \\ Received 1993 March 12; accepted 1993 August 17
}

\begin{abstract}
This paper presents the kinetic equation solution for beam electrons injected during solar flares from the corona to the chromosphere, consisting of hydrogenic plasma with partial ionization. The electrons are considered to lose their energy both in collisional processes with the charged and neutral species of ambient plasma and in ohmic heating by return currents induced in the plasma by beam electrons. The evolution of the energy and angular distributions of energetic electrons is calculated as functions of the column density. The bulk of the electron-beam energy stored in low-energy electrons is shown to be lost in the ambient plasma heating, less via Coulomb collisions at the lower corona and more via ohmic dissipation at the upper chromosphere. More energetic electrons with energies above $120 \mathrm{keV}$ can reach the chromospheric levels with a weak ionized plasma, where a decrease of the Coulomb collisions and the induced electric field of a return current produce beams as well directed as on the top boundary. The X-ray bremsstrahlung polarization is shown to be positive in the range $5 \%-10 \%$. It is very sensitive to the emergent photon energies below $40 \mathrm{keV}$ and to angles of view for all of the $\mathrm{X}$-ray radiation range.

Subject headings: acceleration of particles - plasmas - Sun: flares — Sun: X-rays, gamma rays
\end{abstract}

\section{INTRODUCTION}

Energetic electrons, accelerated to energies from 10 to 300 $\mathrm{keV}$, or thermal fluxes with very high $\left(10^{8} \mathrm{~K}\right)$ kinetic temperatures are suggested to be responsible for the production of $\mathrm{X}$ ray and microwave bursts, extreme ultraviolet (EUV) radiation, and possibly also optical emissions during solar flares. For a correct interpretation of observational data, both their spatial distribution and their temporal correlation in different ranges of electromagnetic radiation, one needs to consider the dispersion in phase space of accelerated electrons. With this approach Leach \& Petrosian (1981) investigated the energetic and angular variations of a distribution of beam electrons injected into a cold hydrogen plasma with the two types of magnetic structure (open and closed), solving the time-independent Fokker-Planck equation, and applied these results to calculations of the influence of the magnetic field inhomogeneity on the degree of X-ray polarization in solar flares (Leach \& Petrosian 1983; Leach, Emslie, \& Petrosian 1985). Haug, Elwert, \& Raussaria (1985) did calculations combining analytically treated multiple-scattering and random large-angle scattering by the Monte Carlo method for initially monoenergetic electrons and found their energy and angular distributions. Nocera, Skrynnikov, \& Somov (1985) solved the kinetic problem for hot thermal electrons with pure Coulomb energy losses with an angular anisotropy in a homogeneous magnetic tube and calculated hard X-ray bremsstrahlung polarization caused by the thermal source.

Return-current energy losses were found to make a noticeable contribution to the injected beam dynamics at the chromospheric level (Emslie 1980), to reduce the penetration distance of the bremsstrahlung-producing electrons, and to

\footnotetext{
${ }^{1}$ Physics Department, Kiev University, Kiev 252022, Ukraine.

${ }^{2}$ Department of Physics and Astronomy, University of Glasgow, Glasgow G12 8QQ, Scotland, UK.
}

increase therefore the total beam energy deposit required (LaRosa \& Emslie 1988; Li 1991). Diakonov \& Somov (1988) solved the kinetic equation for thermal electrons run away from a hot plasma with electron energy losses in both Coulomb collisions and ohmic dissipation via the induced return-current electrical field. For this case the electric field was found to lead to a significant reduction of the convective heat flux carried by these fast thermal electrons due to their return to the source mainly without large energy losses. The hard $\mathrm{X}$-ray bremsstrahlung polarization caused by them was positive and did not exceed 6. In the papers of McClements ( 1991, 1992) an influence of the magnetic field configuration on the temporal evolution of hard X-ray radiation caused by beam electrons was investigated by means of a Fokker-Planck equation solution for a completely ionized ambient plasma with the simultaneous effects of collisions, return currents, and magnetic trapping. The X-ray emission was found to be insensitive to the magnetic field configuration.

All the previous calculations were done on the assumption of a completely ionized hydrogenic plasma and were used mostly for an interpretation of the coronal and transition region emissions where this assumption is correct. On the other hand, in the chromospheric plasma with partial ionization decreasing very fast with depth, inelastic collisions of beam electrons with hydrogen atoms were found to prevail over Coulomb collisions, which causes electron beams injected along the magnetic field direction to be able to penetrate into deeper low chromosphere levels $\left(\xi \geq 10^{20} \mathrm{~cm}^{2}\right.$ ) (Aboudarham \& Henoux 1986; Zharkova \& Kobylinskii 1989, 1993).

This paper presents the kinetic equation numerical solutions for accelerated nonthermal electrons with a power-law distribution in energy and a normal distribution over pitch angles injected during solar flares from the corona to the chromosphere. We take into account both collisional and ohmic losses and find the beam electron distribution functions with their pitch-angle anisotropy over the depth into flaring atmosphere. 
The ambient plasma is assumed to be hydrogenic with partial ionization, so both kinds of collisions-Coulomb collisions and collisions with neutral atoms-are considered. These functions were used for the calculations of the X-ray bremsstrahlung intensities and degrees of polarization observed with different angles of view.

\section{BASIC EQUATIONS}

We suggest the interaction of accelerated electrons with an ambient plasma to be a diffusive process, and therefore the problem is to find the distribution function density of the injected electrons. Let us consider the precipitation of an electron beam from the corona to the chromosphere within a flux tube which is perpendicular to the solar surface. The beam electrons are assumed to lose their energy in collisions with electrons, ions, and neutrals and also via ohmic losses by means of inducing the electrical field of a return current in ambient plasma. The physical parameters of the ambient plasma, such as total density, kinetic temperature, velocity field, and degree of ionization, are given from gasdynamical calculations.

The beam electron distribution function can be found from a solution of the equation (Landau 1937)

$$
v \cos \theta \frac{\delta f}{\delta l}-\frac{e E}{m_{e}} \cos \theta \frac{\delta f}{\delta v}-\frac{e E}{m_{e} v} \sin ^{2} \theta \frac{\delta f}{\delta \cos \theta}=S t(f),
$$

where

$$
\begin{aligned}
S t(f)=\frac{1}{v^{2}} \frac{\delta}{\delta v}\left[v^{2} \nu(\theta)\left(\frac{T_{e}}{m_{e}} \frac{\delta f}{\delta v}\right)\right. & +v f] \\
& +\nu(\theta) \frac{\delta f}{\delta \cos \theta}\left(\sin ^{2} \theta \frac{\delta f}{\delta \cos \theta}\right)
\end{aligned}
$$

is the linearized integral of collisions in the form given by Diakonov \& Somov (1988) with a frequency of collisions between beam electrons and scattering centers of the ambient plasma given by

$$
\nu(\theta)=\frac{k}{\left(2 m_{e}\right)^{1 / 2}} \pi N(\ln \Lambda) e^{4}\left(\frac{m_{e} v^{2}}{2}\right)^{-1.5} .
$$

The variable $k$ is determined by Emslie (1978), $v$ is a beam electron velocity, $\theta$ is the angle between the electron-beam propagation direction and the direction to the Sun's center, $l$ is the linear coordinate, $e$ and $m$ are the electron charge and mass, $E$ is the induced return-current electrical field, and $\ln \Lambda$ is the Coulomb logarithm.

We introduce the following dimensionless variables: $\mu=$ $\cos \theta$ for the angle; $z \equiv m_{e} v^{2} / 2 E_{0}$ for the electron energy, where $E_{0}$ is the lower cutoff energy of accelerated particles; $\epsilon \equiv E_{0} E$ / $\left(2 \pi e^{3} N \ln \Lambda\right)$ for the normalized return-current electric field; $\tau \equiv 2 k T / m_{e} v_{\text {eff }}^{2}$ (small parameter) for the thermal energy; $s \equiv \pi e^{4}(\ln \Lambda) / E^{2} \int N(l) d l$ for the normalized coordinate; and $\Delta_{\mu}$ for the $\mu$-dependent part of the Laplacian differential operator.

In these variables the kinetic equation is transformed to the following:

$$
\begin{aligned}
\mu z^{2} \frac{\delta f}{\delta S}-2 \epsilon \mu z^{2} \frac{\delta f}{\delta z}-\epsilon z(1 & \left.-\mu^{2}\right) \frac{\delta f}{\delta \mu} \\
& =\frac{k}{2}\left(2 \tau z \frac{\delta^{2} f}{\delta z^{2}}+2 z \frac{\delta f}{\delta z}+\Delta_{\mu} f\right) .
\end{aligned}
$$

The induced electric field of a return current was calculated by equating the direct and return electron currents, as was done by McClements (1992), with the classical electroconductivity $\sigma$ multiplied by the integral of velocity over the beam electron distribution functions in the velocity phase space. Thus the kinetic equation (4) becomes a nonlinear integrodifferential one.

\section{METHOD OF SOLUTION}

\subsection{Some Analytical Solutions}

If in equation (4) we take the induced electrical field to be equal to zero, the pure collisional solution $f_{c}$ can be obtained analytically using the method of characteristics. The function $f_{c}$ is the following:

$$
f_{c}=\left[2 \epsilon\left(s-s_{0}\right)+z\right]^{-(\gamma-0.5)},
$$

which is identical to those suggested by Shmeleva \& Syrovatskii ( 1972) and discussed by Zharkova \& Kobylinskii (1992).

Setting the right-hand side of equation (4) to zero, we can find analytically by the same means the pure return-current solution $f_{r}$, which is given by the expression

$$
f_{r}=\left[\frac{4\left(s-s_{0}\right)}{\langle\mu\rangle}+z^{2}\right]^{0.5(-\gamma+0.5)} .
$$

Then, combining these solutions, we estimate the general electron distribution function as the following:

$$
f_{b}=\left(f_{c}+G f_{r}\right) \exp \left[-\frac{\left(\cos ^{-1} \mu-\cos ^{-1}\langle\mu\rangle\right)^{2}}{2 \sigma^{2}}\right] .
$$

The variable $G$ denotes a ratio between these two channels of energy deposit and is described by the expression

$$
G=\frac{z \mu \epsilon}{x+(1-x) \Lambda^{\prime} / \Lambda}
$$

here $x$ is the degree of ionization at a given depth. This solution can be useful in the numerical calculations of equation (4) for estimating the contribution of each of the mechanisms to the electron distributions.

\subsection{Numerical Solutions}

Equation (4) is solved numerically applying the summary approximation method, using the implicit two-level differential schemes with weights on each variable (for details see Samarskii 1989).

A power law in energy and a normal distribution over pitch angles on the top boundary where the beam is injected are 
chosen as an initial condition:

$$
\begin{aligned}
& f^{0}(z, \mu)=A z^{-(\gamma-0.5)} \\
& \quad \times \exp \left[-\frac{\left(\cos ^{-1} \mu-\cos ^{-1}\langle\mu\rangle\right)^{2}}{2 \sigma^{2}}\right],
\end{aligned}
$$

where $A$ is a normalization constant and $\gamma$ is the spectral index of hard X-ray radiation related to the electron-beam spectral index via $\delta=\gamma+1$.

For determination of the region of the energy including ohmic losses and angular variables at a given depth where a solution of the kinetic equation exists, we used equations (13) and (14) from the paper of Emslie (1980), which, in terms of the new dimensionless variables mentioned above, take the following form:

$$
\begin{aligned}
& \frac{d z}{d s}=-\frac{2}{\mu z}\left[x+(1-x) \frac{\Lambda^{\prime}}{\Lambda}\right]-2 \epsilon, \\
& \frac{d \mu}{d s}=-\frac{1}{z^{2}}\left[2 x+(1-x) \frac{\Lambda^{\prime \prime}-\Lambda}{\Lambda}\right]-\frac{1-\mu^{2}}{\mu z} \epsilon,
\end{aligned}
$$

where $\Lambda^{\prime \prime}, \Lambda^{\prime}$, and $\Lambda$ are determined by expressions (8), (12), and (20) in the paper of Emslie. An integration of the equations was carried out by the Runge-Kutta method in a model in which we assume small-angle scatterings $(\Delta \mu / \mu \ll 1)$ and a lack of catastrophic energy losses $(\Delta z / z \ll 1)$. On the edges of the region the distribution functions were accepted in the form of equation (7).

For electrons losing their energy to a limit less than calculated for a region of solution at given depths by equations (10) and (11), a Maxwellian distribution is assumed, and the effects of backscattered electrons (with $\mu<0$ ) on the top boundary are neglected. The last condition of course decreases the number of precipitating electrons at a given depth and may distort a picture of X-ray bremsstrahlung emitted from the corona. But for a study of electrons penetrating into deeper layers, this condition is strong enough, and additional electrons with $\mu<0$ which could precipitate, for instance, in a loop with electron mirroring on the top boundary only enhance the number.

The simulations are fulfilled for the flare models discussed by Somov, Syrovatskii, \& Spektor (1981), where the hydrodynamical response to electron-beam injection was considered. The degrees of ionization were taken from calculations of Zharkova \& Kobylinskii (1989) for the same flare models; they differ from unity beginning from the column density $4 \times$ $10^{19} \mathrm{~cm}^{-2}$ and are equal to $1.5 \times 10^{-2}$ at the density $6 \times 10^{20}$ $\mathrm{cm}^{-2}$. All of the calculations were done for an electron-beam initial energy flux $F_{0}$ at the top boundary equal to $10^{11}$ ergs $\mathrm{cm}^{-2} \mathrm{~s}^{-1}$ and spectral index $\delta=4$ and 6 . We selected the rather low initial energy flux, taking into account the relations between the particle densities and the return current and thermal energies of ambient plasma and electron beam discussed by Diakonov \& Somov (1988) when it is not necessary to consider the wave-particle interactions.

We did calculations for the cutoff energy $E_{1}$ equal to $15 \mathrm{keV}$, and the upper energy limit $E_{2}$ equal to 120,150 , and $250 \mathrm{keV}$. Parameters of the initial pitch-angle distributions were the fol- lowing: $\mu_{\min }=0.5$ (weak directed beam) and $\mu_{\min }=0.7$ (strong directed beam), which define the parameter $\sigma$ in the normal initial angular distribution (5) to be equal to 0.10 and 0.03 , respectively. Using the distribution functions, we calculate the degrees of polarization and intensities of the X-ray bremsstrahlung radiation polarized in parallel and perpendicular directions $\boldsymbol{P}-\boldsymbol{K}$ with regard to the procedure of Nocera et al. (1985), where $\boldsymbol{P}$ is an electron impulse and $\boldsymbol{K}$ is a wavevector of emergent photon, with $\psi$ being the angle between $\boldsymbol{K}$ and a perpendicular to the flux-tube axis, called the "angle of view" (for details see Fig. 4 in Nocera et al. 1985).

\section{RESULTS AND DISCUSSION}

The calculated depth and angular variations of beam electron distribution functions are plotted in Figures 1 and 2, and the hard $\mathrm{X}$-ray bremsstrahlung polarization is plotted against the emergent photon energy for different angles of view in Figure 3.

The solutions of a kinetic equation presented here for electron beams with a power-law energy distribution cannot be compared quantitatively with the similar calculations made for the Fokker-Planck equations by McClements (1991, 1992), as in these papers the electron distribution functions are not plotted. But we can draw some qualitative conclusions on the electron-beam dynamics with depth, comparing these results with previous calculations for initially monoenergetic beams scattering by Coulomb collisions with the pitch-angle anisotropy of Haug et al. (1985) and for pure gyrosynchrotronic losses for the open magnetic field model made in the Fokker-Planck approach by Leach \& Petrosian (1981). Because the authors consider another mechanism of electronbeam energy losses in which contributions vary with depth, their results differ slightly from ours.

Our results include the influence of the two different effects of electron-beam precipitation into flaring atmospheres: return currents and partial ionization. We can use the relation of the energy deposits in these channels ( see expression [7]) like the parameter $\beta$ in Emslie (1980) to understand these contributions qualitatively on each depth. If an ionization is complete (or $x=1$ ), the effect of a return current is absolutely identical to that described by Emslie (1980), LaRosa \& Emslie (1988), and $\mathrm{Li} \mathrm{(1991).} \mathrm{It} \mathrm{appears} \mathrm{in} \mathrm{a} \mathrm{faster} \mathrm{isotropization} \mathrm{of}$ the electron velocity distribution, and as a consequence in a reduced heating of the chromosphere and enhanced heating of the corona. The larger the beam energy flux, the more noticeable is the return-current effect. When the degree of ionization decreases and is close to zero, the effect of a return-current solution is reduced very much by interactions of beam electrons mostly with neutral species of ambient plasma; this is denoted by the factor $\Lambda / \Lambda^{\prime}$. On the other hand, the effect of the pure collisional solution $f_{c}$ in a partially ionized chromospheric plasma investigated in the paper of Zharkova \& Kobylinskii (1993) (see Fig. 2 there) appears in a decrease in the energy deposit rates, which causes a bigger part of the beam electrons to be able to penetrate to deeper layers. So in a partially ionized plasma of the lower chromosphere, we can expect a very essential increase of the number of beam electrons able to reach these depths and, moreover, to produce the additional direct ionization and excitation of neutral hydrogen atoms in such inelastic collisions. 


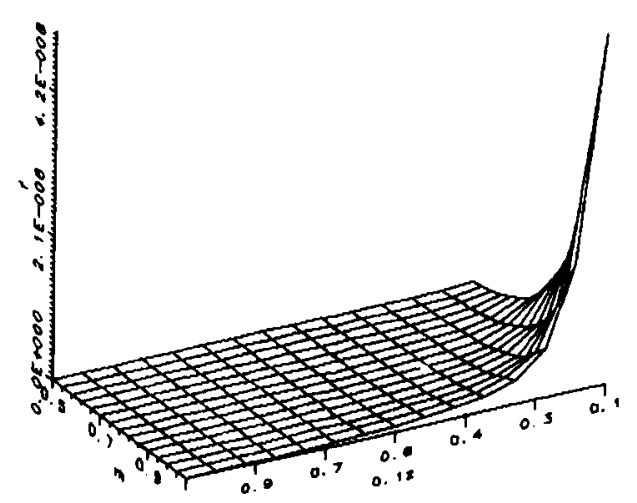

$1(\mathrm{a})$

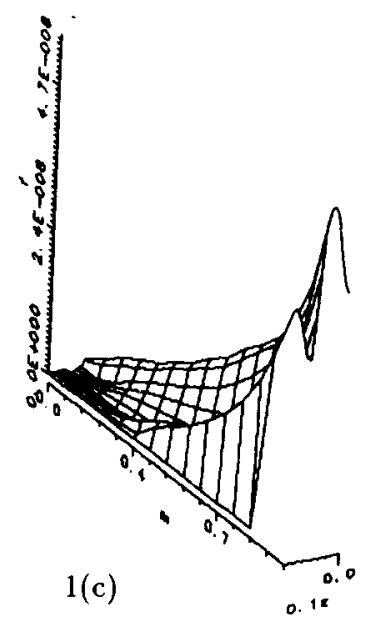

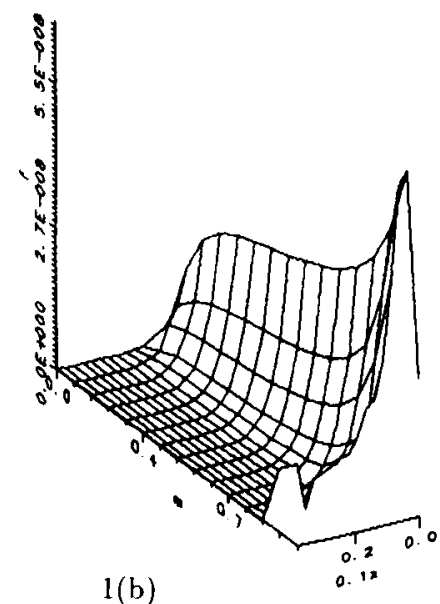

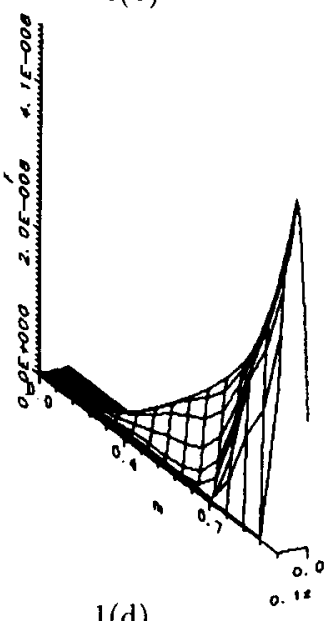

$1(\mathrm{~d})$ FIG. 1.-Beam electron distribution functions in the injection site $(a) s=2.4 \times 10^{17} \mathrm{~cm}^{-2}$, on depths $(b) 3.3 \times 10^{18} \mathrm{~cm}^{-2},(c) 7.6 \times 10^{19} \mathrm{~cm}^{-2}$, and $(d)$
$1.6 \times 10^{20} \mathrm{~cm}^{-2}$ for $\mu_{\min }=0.5$ and the energy range $20-200 \mathrm{keV}$.
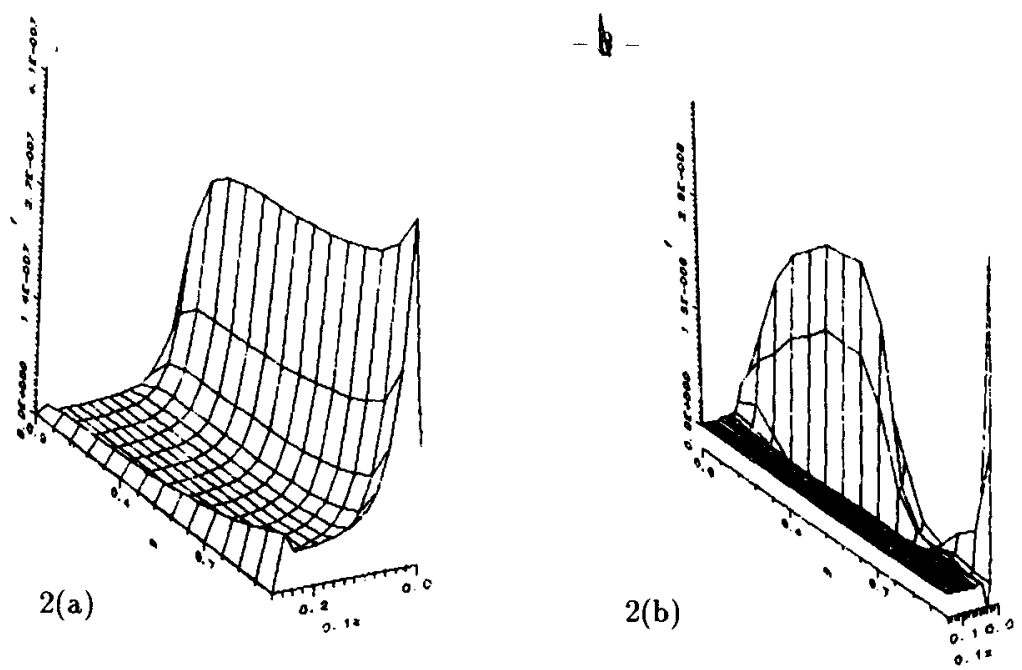

FIG. 2.-Beam electron distribution functions on depths $(a) 3.3 \times 10^{18} \mathrm{~cm}^{-2}$ and $(b) 1.6 \times 10^{20} \mathrm{~cm}^{-2}$ for $\mu_{\min }=0.7$ and the energy range $20-150 \mathrm{keV}$ 


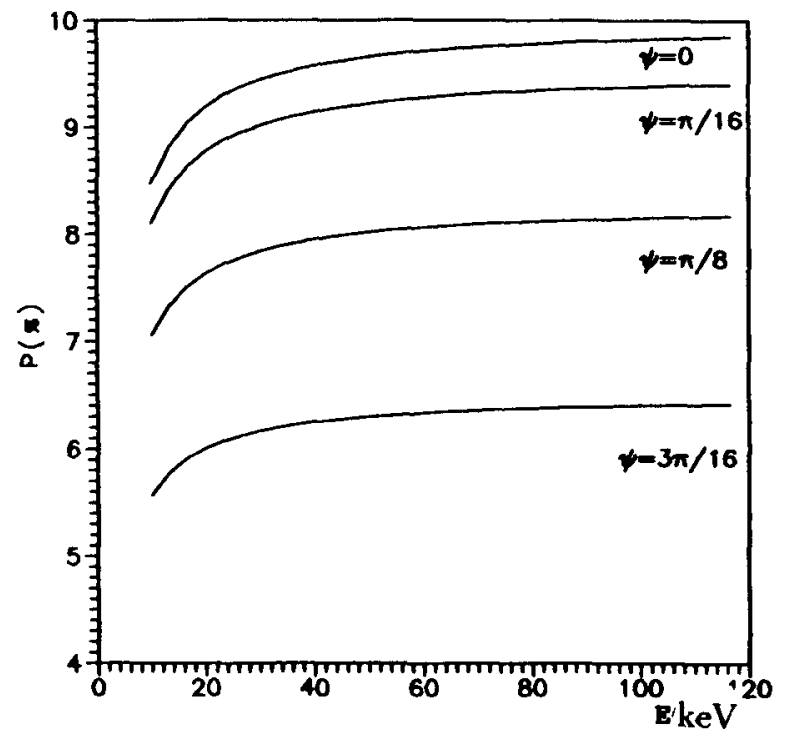

Fig. 3.-X-ray bremsstrahlung polarization $P$ caused by beam electrons with parameters as in Fig. 1 vs. emergent quantum energies for the different angles of view $\psi$.

This is justified by present calculations of the beam electron distribution functions, which are shown to decrease smoothly and uninterrupted throughout all depths of the solar atmosphere. At some depths divisions of initial beams on pitch angles and energies can be seen. Electron beams with the upper energy limit $E_{2} \leq 120 \mathrm{keV}$ are braking by collisions and the induced electrical field of a return current and move in the opposite direction ( pitch angles close to $90^{\circ}$ ), with a wide normal distribution at levels before the electron stopping depth $\left(\xi \simeq 2 \times 10^{19} \mathrm{~cm}^{-2}\right.$ ) where the ionization is still complete ( see Fig. 2). The similar effect of the beam electron isotropization on pitch angles was obtained by Haug et al. (1985) even for initially monoenergetic electrons, and by Leach \& Petrosian (1981) for the open magnetic field model, but their depths were much bigger than those found in our calculations. Including additional channels of energy losses in scattering with the pitch-angle anisotropy results in much faster production of the beam electrons moving in different directions, and with a return current the induced electrical field will direct their motion away from a source. This resembles a return to the source of thermal electrons affected by an induced electric field, discussed by Diakonov \& Somov (1988).

Another kind of initial beam division (on the energy see Figs. $1 b$ and $1 c$ ) which appears at the transition region is suggested to be a result of the Kelvin-Helmholtz two-beam instability, and it disappears at deeper layers where a return beam becomes negligible.

After such a division on pitch angles, beams with upper energy limits more than $120 \mathrm{keV}$ move into deeper levels, and at greater depths $\left(>10^{20} \mathrm{~cm}^{-2}\right)$ they again become single beams directed along the flux-tube axis (Fig. 1d), but their densities at these levels sharply depend on the beam upper energy limit. The higher the upper energy, the higher the beam density at these depths. Apparently, after a loss of the low-energy beam particles at the coronal and transition region (TR) levels, the higher energy electrons of beams move in ambient plasma with partial ionization, and lose their energy mainly in collisions with neutral atoms, not scattering with such an angular anisotropy as with charged particles, that produce beams as well directed as on the top boundary. These beams can therefore penetrate to deeper layers of the solar chromosphere and even the photosphere, and this is in good agreement with the conclusions of Aboudarham \& Henoux (1986) and Zharkova \& Kobylinskii ( 1993) deduced from the interpretation of the hydrogen emission in the impulsive solar flares.

The influence of a width of the anisotropic injection via $\mu_{\min }$ appears in the increase of the upper energy limit to $150 \mathrm{keV}$ for beam electrons able to reach the deeper layers, i.e., the thermalization rates for these electrons are bigger (compare Figs. 1 and 2).

The above beam electron distribution functions were tested by calculations of the X-ray bremsstrahlung intensities and degrees of polarization (Fig. 3). The polarization observations are not very numerous and were reported for X-rays with a moderate hardness earlier by Somov \& Tindo (1978) to be rather high (up to $40 \%$ ) and later by Tramiel, Chanan, \& Novick (1984) to be lower $(5 \%-12.5 \%)$. The observations were discussed by Leach et al. (1985), where the authors, using the results of the Fokker-Planck model of Leach \& Petrosian (1981), have computed the degrees of polarization as a function of the angle of view and found them to be very small $(<16$ $\mathrm{keV}$ ); electrons of a different directionality and hardness can produce such wide differences in the polarization.

Here we calculated the polarization for hard X-rays with emergent photon energies from 10 to $300 \mathrm{keV}$ for different angles of view caused by beam electrons with energies 15-250 $\mathrm{keV}$ and spectral index 4 . Our calculations give the hard $\mathrm{X}$-ray polarization to be in the range $5 \%-10 \%$ (Fig. 3), which is higher than reported by Leach et al. ( 1985) and is still quantitatively in very good agreement with the observations of Tramiel et al. (1984), taking into account that there is not a spatial orientation of the observations. The degrees are shown to increase sharply for the emergent photon energies $E_{2} \leq 40 \mathrm{keV}$ and to be almost constant for higher energies, and to show more noticeable dependences on the angle of view $\psi$, with maximum values for $\psi=0$ and decreasing with increasing $\psi$. Because even a variety of conditions of the observations is able to produce the observed polarization variations, for a comprehensive fitting of observed polarization data one needs more accurate observations of the X-ray radiation and hard X-ray solar images of good quality for the loop geometry and fieldline orientation to be obtained.

It would be interesting to apply our results to a model with the magnetic field and electron mirroring and to study the joint effect on the beam dynamics in a partially ionized plasma; we plan to do this in a forthcoming paper.

We would like to express our deepest appreciation to the Royal Society of London for the award to V. V. Z. of a Research Fellowship and for allowing us this opportunity to report our results at IAU Colloquium 142. We would like to thank the Scientific Organizing Committee, and to personally thank E. Chupp and M. Chupp for their inestimable help with the presentation of this paper. Also, we thank the referee for his very constructive comments, from the inclusion of which the paper has only benefited. 
Aboudarham, J., \& Henoux, J. C. 1986, A\&A, 156, 73

Diakonov, S. V., \& Somov, B. V. 1988, Sol. Phys., 116, 119

Emslie, A. G. 1978, ApJ, 224, 241 1980, ApJ, 235, 1055

Haug, E., Elwret, G., \& Rausaria, R. R. 1985, A\&A, 148, 115

Landau, L. D. 1937, Zh. Eksper. Teoret. Fiz., 7, 203

LaRosa, T. N., \& Emslie, A. G. 1988, ApJ, 326, 997

Leach, J., Emslie, A. G., \& Petrosian, V. 1985, Sol. Phys., 96, 331

Leach, J., \& Petrosian, V. 1981, ApJ, 251, 781

- 1983, ApJ, 269, 715

Li, P. 1991, Ph.D. thesis, Univ. Alabama, Huntsville

McClements, K. G. 1991, Vistas Astron., 34, 325

\section{REFERENCES}

McClements, K. G. 1992, A\&A, 258, 542

Nocera, L., Skrynnikov, Ju. I., \& Somov, B. V. 1985, Sol. Phys., 97, 81

Samarskii, A. A. 1989, Differential Scheme Theory (Moscow: Nauka), 616

Shmeleva, O. P., \& Syrovatskii, S. I. 1972, Soviet Astron.-AJ, 49 (No. 2), 145

Somov, B. V., Syrovatskii, S. I., \& Spektor, A. R. 1981, Sol. Phys., 73, 145

Somov, B. V., \& Tindo, I. P. 1978, Cosmic Res., 16, 555

Tramiel, L. J., Chanan, G. A., \& Novick, R. 1984, ApJ, 280, 440

Zharkova, V. V., \& Kobylinsky, V. A. 1989, Soviet Astron. Lett., 15, 366 1992, Kinem. Phys. Terrest. Bodies, 32, 45 . 1993, Sol. Phys., 143, 259 